\title{
FUNCTION PROJECTIVE SYNCHRONIZATION OF NEW CHAOTIC REVERSAL SYSTEMS
}

\author{
Olurotimi S. Ojoniyi \\ Department of Physics and Telecommunications, Tai Solarin University of Education, \\ Ijagun, Ogun State Nigeria.
}

\begin{abstract}
In the present work, Lyapunov stability theory, nonlinear adaptive control law and the parameter update law were utilized to derive the state of two new chaotic reversal systems after being synchronized by the function projective method. Using this technique allows for a scaling function instead of a constant thereby giving a better method in applications in secure communication. Numerical simulations are presented to demonstrate the effective nature of the proposed scheme of synchronization for the new chaotic reversal system.
\end{abstract}

\section{KEYWORDS}

Function projective synchronization, Lyapunov stability theory, chaotic system, new chaotic reversal system.

\section{INTRODUCTION}

The chaotic dynamics observed only in nonlinear systems have been largely defined by oscillations which are sensitive to initial conditions [1]. Lorenz and Rossler systems are pioneering simple chaotic systems discovered with a lot of interesting properties that can model physical systems [1]-[2]. This relatively new dynamical behaviour has since been discovered in other disciplines such as engineering, science and economics. In order to understand fully most nonlinear phenomenon in nature through interaction of systems, the control and synchronization of chaotic oscillations is vital.

The process of controlling and synchronising chaotic systems has attracted much attention since its discovery in less than twenty years [3]. The work of Pecora and Caroll [4] generated a wider research in synchronization since it entails the synchronization of two identical chaotic systems with different initial conditions.A number of methods of synchronization have been proposed since the pioneering work of Pecora and Caroll such as complete synchronization, generalized synchronization, phase synchronization, lag synchronization, adaptive synchronization, timescale synchronization, intermittent synchronization, projective synchronization and function projective synchronization [5]-[9]. Function projective synchronization deserves much attention since it has been found applicable and better in secure communication.

Function projective synchronization implies that the master and slave oscillators could be synchronized up to a scaling function unlike a constant in the projective synchronization. The 
advantage is that it becomes more complex to determine the function used and as a result more secure communication is achieved under this scheme.

In the present work, function projective synchronization of new chaotic reversal systems [10] with uncertain parameters using nonlinear adaptive controller has been investigated to demonstrate the effectiveness of this scheme as it applies in secure communication. The remaining part of this work is arranged as follows: section 2 is on the nonlinear adaptive controller designed to synchronize the new chaotic reversal systems, section 3 is on numerical simulations presented to demonstrate the robust nature of the scheme, and section 4 is the conclusion of the paper.

\section{ADAPTIVE CONTROL SCHEME}

In the present section, we examine briefly the dynamics of the new deterministic model for chaotic reversal [10].

We have a new deterministic model for chaotic reversal by C. Gissinger [10] given by:

$$
\begin{array}{r}
\dot{x}_{1}=a x_{1}-x_{2} x_{3} \ldots \ldots \ldots . \\
\dot{x}_{2}=-b x_{2}+x_{1} x_{3} \\
\dot{x}_{3}=c-x_{3}+x_{1} x_{2}
\end{array}
$$

Where $x_{1}, x_{2}$ and $x_{3}$ are the states and $a, b, c$ are unknown parameters.

The system (1) is chaotic when the parameter values are taken as:

$a=0.119, b=0.1$ and $c=0.9$ as shown in figure 1.

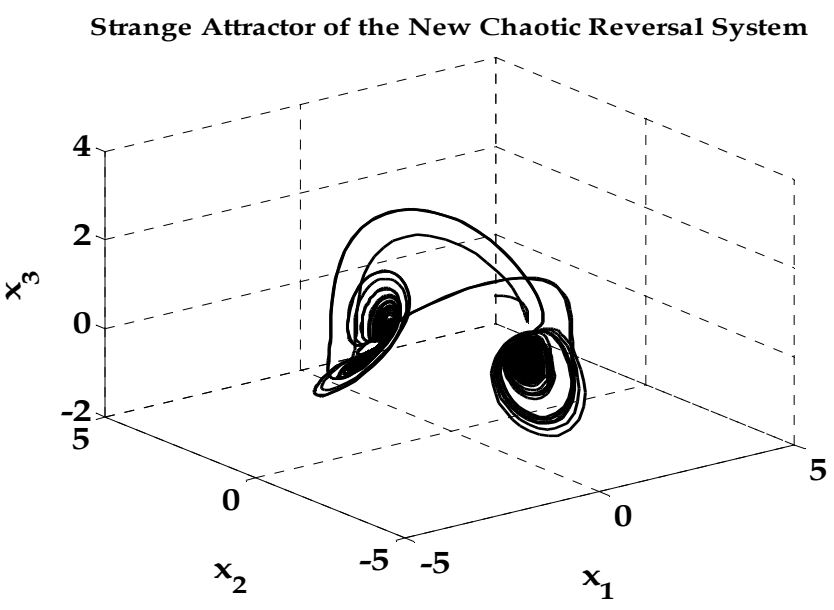

Figure 1:

The system (1) is taken as master system, and the slave system with the adaptive control scheme is taken as

$$
\begin{array}{r}
\dot{y}_{1}=a_{1} y_{1}-y_{2} y_{3}+u_{1} \ldots \ldots . . \\
\dot{x}_{2}=-b_{1} y_{2}+y_{1} y_{3}+u_{2}
\end{array}
$$


International Journal of Computer Science, Engineering and Information Technology (IJCSEIT), Vol. 4, No.5, October 2014

$$
\dot{x}_{3}=c_{1}-y_{3}+y_{1} y_{2}+w_{3}
$$

Where $a_{1}, b_{1}, c_{1}$ are the parameters of the slave which needs to be estimated and $u_{1}, u_{2}, u_{3}$ are the nonlinear controllers such that the two new chaotic reversal systems are function projective synchronized in the sense that

$$
\lim _{t \rightarrow \infty}\left\|y_{i}-\alpha(t) x_{i}\right\|=0, i=1,2,3
$$

Where $\alpha(t)$ is the scaling function.

From system (1) and (2) we get the error dynamical system which can be written as

$$
\begin{gathered}
b_{1}=a_{1} y_{1}-y_{2} y_{3}+u_{1}-\alpha(t)\left(a x_{1}-x_{2} x_{3}\right)-d(t) x_{1} \ldots \ldots \ldots \\
e_{2}=-b_{1} y_{2}+y_{1} y_{3}+u_{2}-\alpha(t)\left(-b x_{2}+x_{1} x_{3}\right)-\alpha(t) x_{2} \\
b_{3}-c_{2}-y_{3}+y_{1} y_{2}+u_{3}-\alpha(t)\left(c-x_{3}+x_{2} x_{2}\right)-\alpha(t) x_{3} \\
\text { Where } a_{2}(t)=y_{2}(t)-\alpha(t) x_{2}(t) \quad i=1,2,3
\end{gathered}
$$

In order to stabilize the error variables of system (3) at the origin, we propose the adaptive control law and the parameter update law for system (3) as follows

$$
\begin{gathered}
u_{1}=-a_{1} y_{1}+y_{2} y_{3}+\alpha(t)\left(a_{1} x_{1}-x_{2} x_{3}\right)+d(t) x_{1}-k_{1} e_{1} \ldots \ldots(5) \\
u_{2}=b_{1} y_{2}-y_{1} y_{3}+\alpha(t)\left(-b_{1} x_{2}+x_{2} x_{2}\right)+\alpha(t) x_{2}-k_{2} e_{2} \\
u_{3}=-c_{1}+y_{3}-y_{1} y_{2}+\alpha(t)\left(c_{1}-x_{3}+x_{1} x_{2}\right)+\alpha(t) x_{3}-k_{3} e_{3}
\end{gathered}
$$

And the update rule for the three uncertain parameters $a_{1}, b_{1}, c_{1}$, are

$$
\begin{gathered}
d_{1}=x_{1} \alpha(t) e_{1}-k_{4} e_{a} \ldots \ldots . . . \\
b_{1}=x_{2} \alpha(t) e_{2}-k_{5} e_{b} \\
\dot{c}_{1}=a(t) e_{3}-k_{6} e_{c}
\end{gathered}
$$

Where $k_{i}>0(i=1,2,3, \ldots .6)$ and $e_{a}=a_{1}-a, e_{b}=b_{1}-b_{,} e_{d}=c_{1}-c$,

Theorem For the given scaling function $\alpha(t)$, the function projective synchronization between the master system (1) and the slave system (2) can be achieved if the nonlinear controller (5) and the update law (6) are adopted.

Proof We construct the following Lyapunov function

$V=\frac{1}{2} \sum_{i=1}^{3} e_{i}^{T} e_{i}+\frac{1}{2}\left(e_{a}^{2}+e_{b}^{2}+e_{a}^{2}\right)$

Calculating the time derivative of $\mathrm{V}$ along the trajectory of error system (4), we have 


$$
\begin{aligned}
& \dot{V}=e_{1}(t)\left[-a_{1} y_{1}+y_{2} y_{3}+u_{1}-\alpha(t)\left(-\alpha x_{1}+x_{2} x_{3}\right)-\alpha(t) x_{1}\right] \\
& +s_{2}(b)\left[-b_{1} y_{2}+y_{1} y_{3}+u_{2}-u(b)\left(-b x_{2}+x_{1} x_{3}\right)-\dot{u}(b) x_{2}\right] \\
& +e_{3}(t)\left[c_{1}-y_{3}+y_{1} y_{2}+u_{3}-\alpha(t)\left(c-x_{3}+x_{1} x_{2}\right)-d(t) x_{3}\right] \\
& \left.\left.+e_{a}(t)\left[x_{1} \alpha(t) e_{1} \quad k_{4} e_{a}\right] \mid \begin{array}{lll}
e_{b}(t)\left[x_{2} \alpha(t) e_{2}\right. & k_{5} e_{b}
\end{array}\right] \mid \begin{array}{ll}
e_{g}(t)\left[\alpha(t) e_{3}\right. & k_{6} e_{c}
\end{array}\right] \\
& =-e^{\tau} K e
\end{aligned}
$$

Where $\left.e=\left[e_{1}, e_{2}, e_{3}, e_{a}, e_{b}, e_{c}\right]\right]^{T}$, and $\boldsymbol{K}=\operatorname{diag}\left[k_{1}, k_{2}, k_{3}, k_{4}, k_{5}, k_{6}\right]$.

It is evident that $\bar{V}$ is negative definite and $\bar{V}=0$ if and only if $e(t)=0$. By the Lyapunov stability theory, the function projective synchronization is achieved. This ends the proof.

\section{NUMERICAL SIMULATIONS}

The numerical simulations to verify the effectiveness of the proposed function projective synchronization using the adaptive controllers are carried out by the fourth-order Runge-Kutta method. This method is used to solve the master system (1) and the slave system (2) with time step size $10^{-6}$. The initial conditions of the master system are $x_{1}(0)=1, x_{2}(0)=2, x_{3}(0)=3$, the slave system are $y_{1}(0)=2, y_{2}(0)=-4, y_{3}(0)=$ 5 , and parameter estimates are $a_{1}(0)=0.02, b_{1}(0)=0.03, d_{1}(0)=0.08$

. The scaling function is chosen as $\sin (t)+\cos (t)$ and $k_{i}=0.5$, for $i=1,2,3, \ldots .6$. The figure 2 shows that the error variables $e_{2}, e_{2}, e_{3}$ tend to zero with $t \rightarrow \infty$. Figure 3 shows that the estimated values of the uncertain parameters converge to $a=0.119, b=0.1, c=0.9$ as $t \rightarrow \infty$.

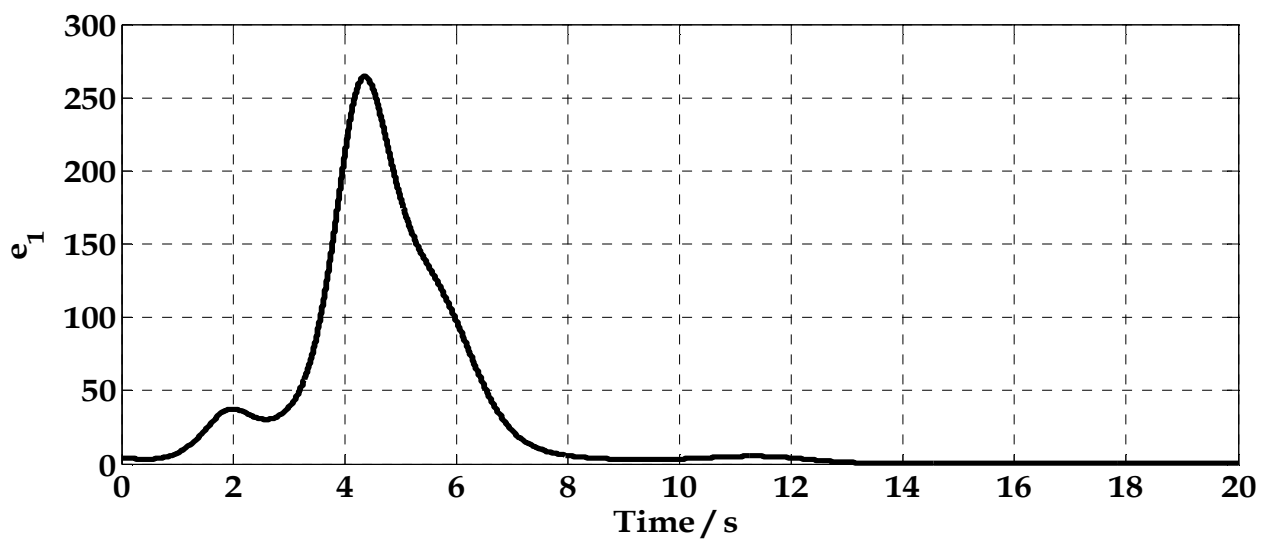


International Journal of Computer Science, Engineering and Information Technology (IJCSEIT), Vol. 4, No.5, October 2014
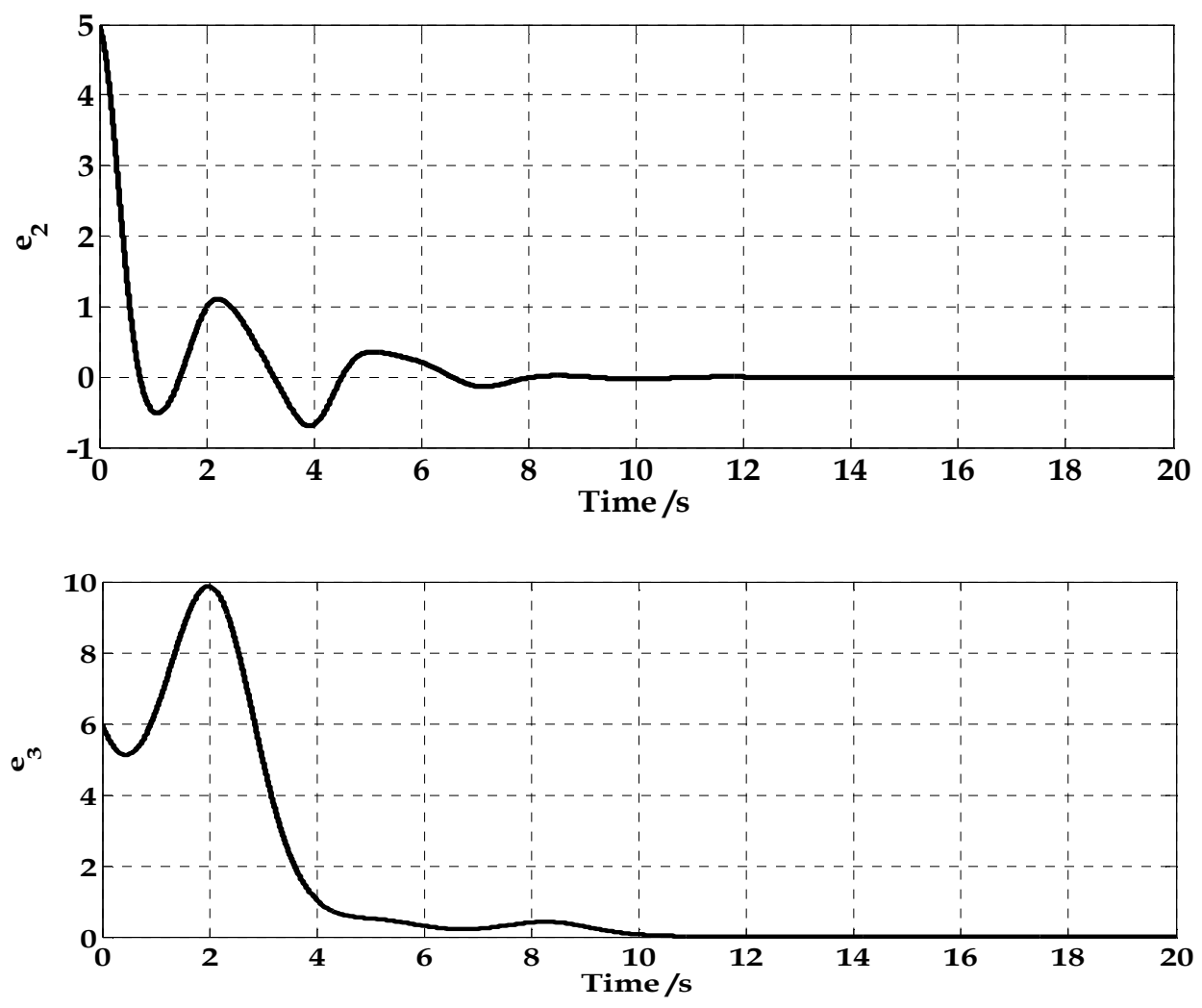

Figure 2: Error dynamics between master system (1) and the slave system (2)

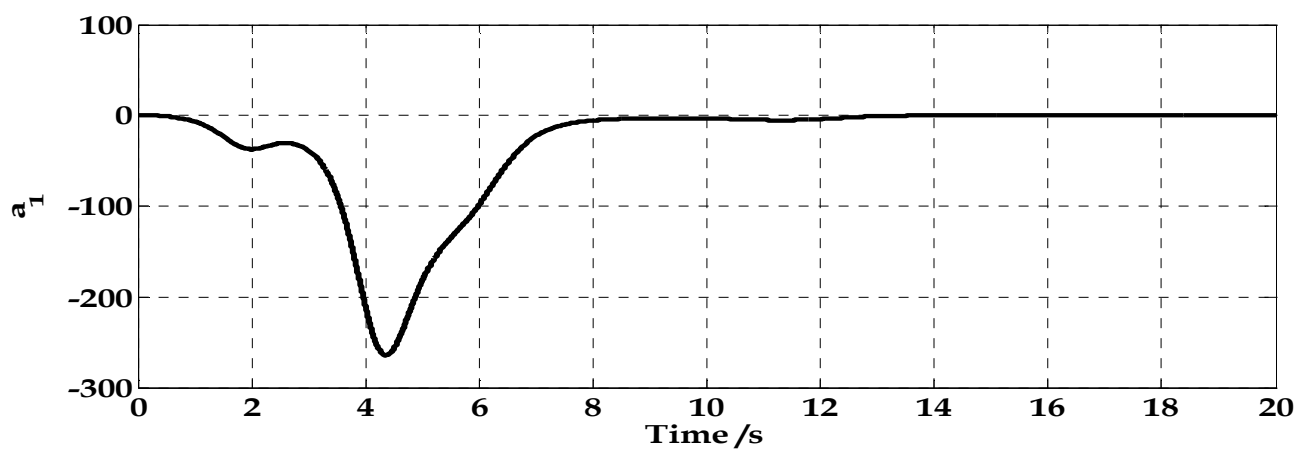


International Journal of Computer Science, Engineering and Information Technology (IJCSEIT), Vol. 4, No.5, October 2014
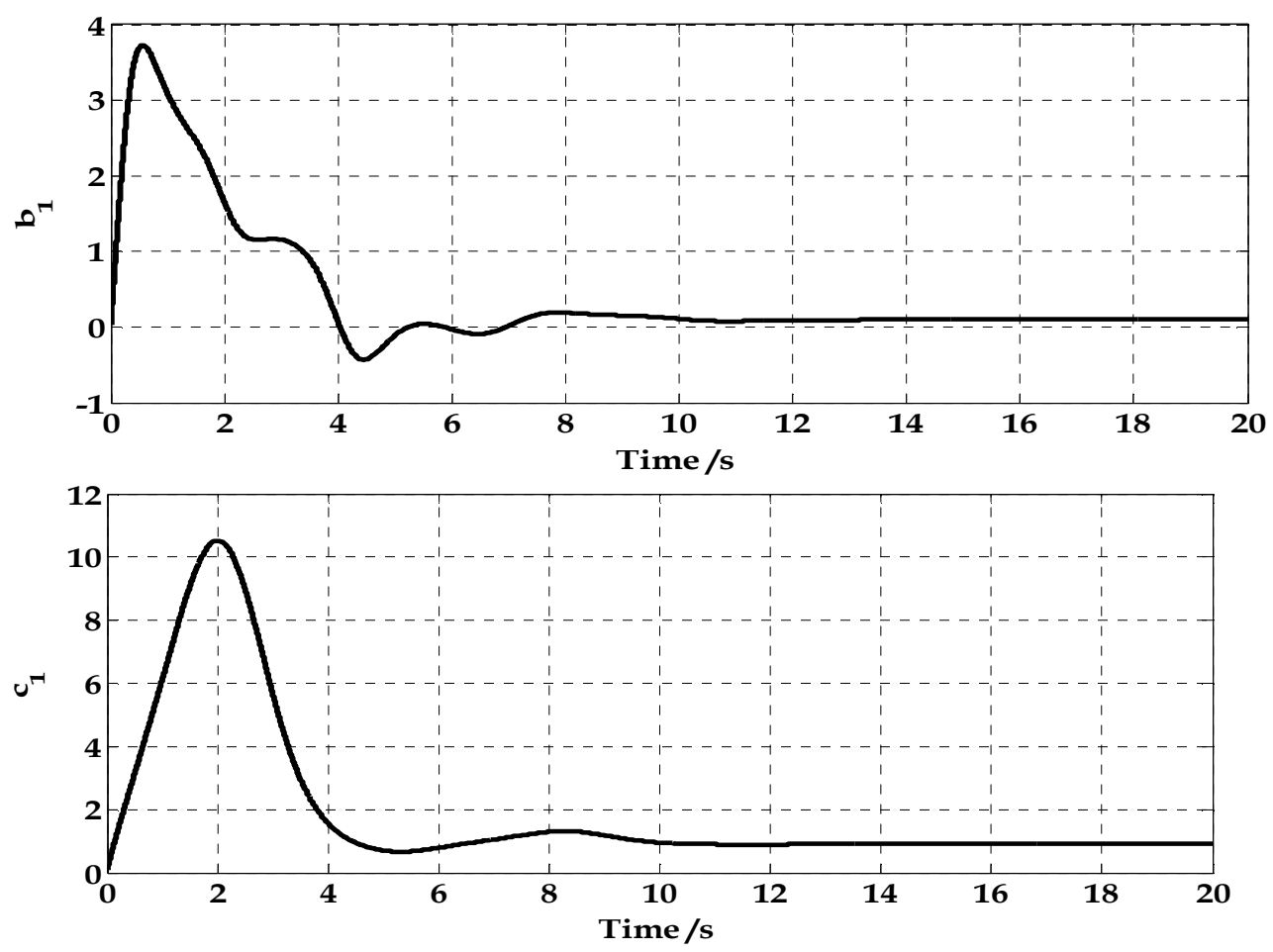

Figure 3: The time evolution of the estimated parameters

\section{CONCLUSION}

In the present work we have demonstrated the application of function projective synchronization in synchronizing the new chaotic reversal systems with uncertain parameters. The Lyapunov stability theory was used to design the adaptive synchronization controllers with associated parameter update laws to stabilize the error dynamics between the master and the slave chaotic oscillators. All the theoretical investigations have been verified by numerical simulations to proof the effectiveness of the scheme.

\section{REFERENCES}

[1] Lorenz, E.N. (1963) “Deterministic nonperiodic flow,” J. Atmos. Phys. Vol. 20, pp 131-141.

[2] Rössler, O.E. (1976) “An equation for continuous chaos,” Physics Letters A, Vol. 57, pp 397-398.

[3] Junbiao Guan, (2012 ) "Function projective synchronization of a class of chaotic systems with uncertain parameters', Hindawi Publishing Corp., Article ID 431752,

[4] L. M. Pecora and T. L. Carroll, (1990) "Synchronization in chaotic systems," Physical Review Letters, vol. 64, no. 8, pp. 821-824,.

[5] M. Rosenblum, A. Pikovsky, and J. Kurths, (1996) "Phase synchronization of chaotic oscillators," Physical Review Letters, vol. 76, pp. 1804-1807,.

[6] H. Taghvafard and G. H. Erjaee, (2011)"Phase and anti-phase synchronization of fractional order chaotic systems via active control," Communications in Nonlinear Science and Numerical Simulation, vol. 16, no. 10, pp. 4079-4088,.

[7] R. Mainieri and J. Rehacek, (1999)"Projective synchronization in three-dimensional chaotic systems," Physical Review Letters, vol. 82, pp. 3042-3045,. 
[8] Junbiao Guan, (2010) "Synchronization control of two different chaotic systems with known and unknown parameters," Chinese Physics Letters, vol. 27, Article ID 020502,.

[9] L. Runzi, (2008) "Adaptive function project synchronization of Rossler hyperchaotic system with uncertain parameters," Physics Letters. A, vol. 372, no. 20, pp. 3667-3671,

[10] Gissinger, C. (2012) ' A new deterministic model for chaotic reversals, ' Euro. Phy. J. B 85:137, pp 1-12.

\section{Author}

Ojoniyi, Olurotimi Seyi is currently on a Ph.D programme in nonlinear dynamics and chaos at the department of Physics of the University of Agriculture, Abeokuta, Ogun State, Nigeria. He holds a M.Sc, degree in Physics from the University of Ibadan, Ibadan, Nigeria and is currently an academic Staff of Physics and Telecommunications department of Tai Solarin University of Education, Ijagun, Ogun State, Nigeria.

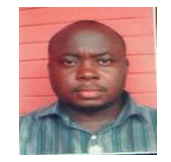

